\title{
Data analysis of low frequency transmitter signals received at a midlatitude site with regard to planetary wave activity
}

\author{
E. D. Schmitter \\ University of Applied Sciences Osnabrueck, 49076 Osnabrueck, Germany \\ Correspondence to: E. D. Schmitter (e.d.schmitter@hs-osnabrueck.de)
}

\begin{abstract}
More than $2 \mathrm{yr}$ of continuously recorded signal amplitude data from the MSK transmitters NRK/TFK $(37.5 \mathrm{kHz}$, Iceland) and NSY $(45.9 \mathrm{kHz}$, Sicily) received at $\left(52^{\circ} \mathrm{N} 8^{\circ} \mathrm{E}\right)$ in the time range from August 2009 to September 2011 are analyzed with regard to planetary wave activity. Wavelet analysis of the day/night amplitude ratio reveals clear evidence of quasi 16 day periods mainly during winter time as well as traces of 5 and 10 day periods on both paths. The amplitude ratio is well correlated to the typical stratospheric $(10 \mathrm{hPa})$ seasonal temperature profile - more clearly to be seen on the northern path. The results are in line and an extension of manifold research with regard of ionospheric absorption phenomena caused by atmospheric wave activity. Continuous monitoring of transmitters in the $40 \mathrm{kHz}$ frequency range proved as an inexpensive tool for investigating mesospheric response to forcing from below.
\end{abstract}

\section{Introduction}

Remote monitoring of lower ionosphere conditions using low and very low transmitters signal propagation is a well known method for several decades. Especially MSK (Minimum Shift Keying) transmitters prove as useful in this respect because of their constant amplitude emissions. For several years we have analysed the signal amplitude variations of 2 transmitters, NRK/TFK $\left(37.5 \mathrm{kHz}, 63.9^{\circ} \mathrm{N} 22.5^{\circ} \mathrm{W}, \mathrm{L}=5\right.$, Iceland) and NSY $\left(45.9 \mathrm{kHz}, 37.13^{\circ} \mathrm{N} 14.44^{\circ} \mathrm{E}, \mathrm{L}=1.4\right.$, Sicily) received a midlatitude site $\left(52^{\circ} \mathrm{N} 8^{\circ} \mathrm{E}\right)$. The NW path to Iceland is particularly suited to study lower ionosphere forcing from above (especially auroral particle precipitation, Schmitter, 2010), whereas both paths yield useful information about forcing processes from below. In Schmitter (2011) we presented data analysis and propagation modeling of the southern path to Sicily. In this paper we extend the analy- sis with respect to forcing from below to the northern path and use wavelet analysis for period identification. A lot of research has been done with respect to the coupling of atmospheric phenomena (tidal, gravity and planetary waves) to the ionosphere and an important remote sensing tool in this respect is lower ionospheric absorption of radio wave propagation between ground based transmitters and receivers (Schwentek, 1974; Fraser, 1977; Lastovicka et al., 1994b; Lastovicka, 1994). Our work is based on measurements using $40 \mathrm{kHz}$ transmitters near to the very low frequency range $(3-30 \mathrm{kHz})$ at a distance of around $2000 \mathrm{~km}$. Propagation at this frequency is sensitive to the conditions in the lowest part of the ionosphere (around 65 to $85 \mathrm{~km}$ ) whereas most of the cited work uses long and medium frequencies (some hundred $\mathrm{kHz}$ to a few $\mathrm{MHz}$ ) penetrating further into the ionosphere. The essential parameters of the lower ionosphere, i.e. electron density and collision frequency, are modulated by atmospheric waves ascending from tropospheric and stratospheric heights to the mesosphere (Lauter et al., 1984; Lastovicka et al., 1994a).

Planetary waves are disturbances having zonal wavelengths of the scale of the earth's radius. Excited mainly by orographic and land-sea temperature contrasts a broad spectrum of planetary waves is generated. The cavity properties of the atmosphere then allow for mainly westward travelling normal modes with periods near to $2,5,10$ and 16 days to propagate into the stratosphere and mesosphere. For a thorough introduction into the topic see Volland (1988). By coupling to the ionosphere they can effect radio wave propagation through modulation of the electron density and neutral density profiles. The latter profile controls the electron collision frequency. Also their variability is suggested to be a signature of possible climatic changes (Pogoreltsev et al., 2009). In following we shortly describe the used wavelet data analysis method and afterwards we discuss results of the last two years of monitoring (August 2009 to September 2011). 


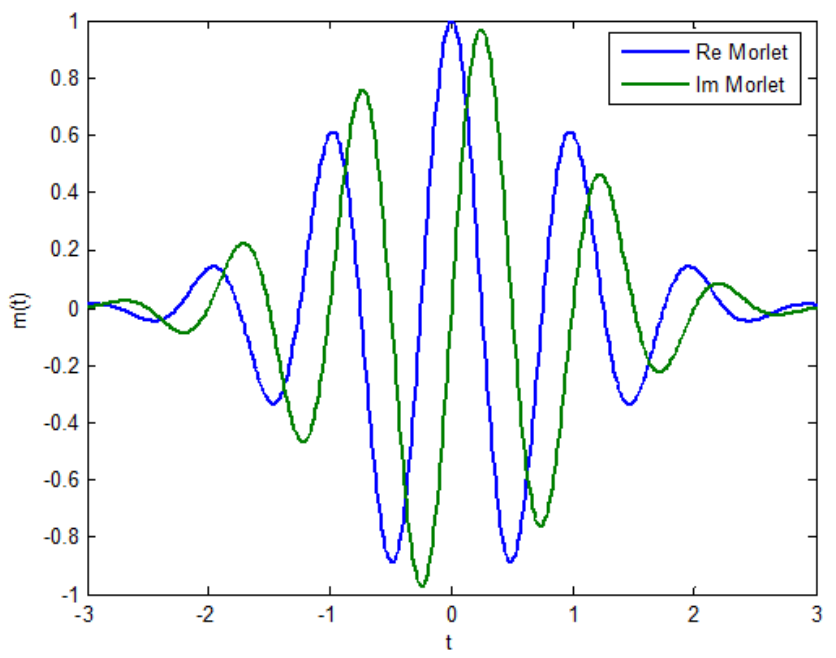

Fig. 1. Morlet wavelet: wave packet with real and imaginary part. The std. deviation of the Gaussian envelope function represents the time domain scaling period $s$. Its Fourier transform is a real Gaussian acting like a bandpass with center at $f=1 / s$ in the frequency domain.

\section{Data analysis}

For period identification we use a wavelet transformation $W$ of the data $y(t)$

$W[y](s, t)=\frac{1}{s} \int_{-\infty}^{+\infty} y(z) m\left(\frac{1}{s}(z-t)\right) \mathrm{d} z$

of the Morlet type (Goupillaud et al., 1984) with: $m(t)=$ $e^{-t^{2} / 2} e^{2 \pi i t}$. In the time domain the scaled Morlet $m(t / s)=$ $e^{-t^{2} / 2 s^{2}} e^{2 \pi i t / s}$ is a complex valued wave packet (Fig. 1) with width $2 s$ (more precisely: $s$ is the standard deviation of the Gaussian envelope function). In the frequency domain its Fourier transform $F$ acts like a band pass filtering out the scaling period $s: F[m(t / s)](\omega)=e^{-(\omega-2 \pi)^{2} s^{2} / 2}$ with a maximum at $\omega=2 \pi f=2 \pi / s$ or $f=1 / s$ and a full width at half maximum of $\Delta f=0.375 / s$. The ripple spacings in the Morlet periodograms shown in this paper reflect the period $s$ indicated on the vertical axis, respectively.

The upper part of Fig. 2 displays the zonally averaged temperature at the 10 mbar level (about $30 \mathrm{~km}$ height) between 65 and 25 degree northern latitude (data from ftp.cpc.ncep. noaa.gov) and its wavelet decomposition. Both radio propagation paths are in this latitude range.

Figure 2, lower part, and Fig. 3, upper part, show the signal amplitude ratio noon/midnight ( $2 \mathrm{~h}$ averages) and wavelet decompositions for the NRK and NSY propagation paths to $52^{\circ} \mathrm{N} 8^{\circ} \mathrm{E}$. A ratio of $0 \mathrm{~dB}$ indicates equal average amplitudes at noon and midnight, whereas negative values mainly result from reduced noon amplitudes and indicate increased day over absorption. We see strong absorption events with large variability during northern winter time (October to
April) and significantly reduced absorption and variability during summer (May to July). These features are more prominently to be seen on the northern propagation path (NRK, Iceland to $52^{\circ} \mathrm{N} 8^{\circ} \mathrm{E}$ ) but also evident on the southern path (NSY, Sicily to $52^{\circ} \mathrm{N} 8^{\circ} \mathrm{E}$ ). During summer time the NRK (northern path) absorption is low and exhibits few variation and correlates well with the zonally averaged $10 \mathrm{mb}$ temperature course. The NSY (southern path) exhibits significant absorption variation also during summer time and the difference of the averaged day/night signal ratio between winter and summer is less pronounced than with the northern path. On the northern path we additionally see a low variation level around $-5 \mathrm{~dB}$ during mid of August to end of September. With both radio signals as well as the stratosphere temperature we see common maxima around June and July with low variation and periods of high variability during winter. We relate the variability to planetary wave activity: the wavelet decompositions of the radio signal time series clearly show 12 to 17 days periods during winter time on both paths, more intensely during winter 2009/2010 than during winter 2010/2011. In fact there are also traces of periods near 2, 5 and 10 days in both radio wavelet decompositions, however not showing up in the cross correlation of the two radio time series, in contrast to the near 16 day period. So with some caution we suggest that normal planetary wave modes below 16 days can be also identified in the propagation data, however not in timely coincidence. The commonality of the near 16 day periodicities is highlighted by the wavelet cross correlation between the NRK and NSY time series shown in Fig. 4 (top). Plotted is the absolute value of the product of the two single wavelet periodograms. The 7 day periods are caused by weekly drop outs of the transmitters. In the middle of Fig. 4 we see the product of the two radio data wavelet periodogramms and the zonal temperature periodogramm (3fold cross correlation). Prominent are the near 16 day period correlations during winter 2009/2011 between the two propagation paths as well as with the temperature data. Less significant but nevertheless clear are the near 13 and 18 day period correlations during winter 2010/2011.

We have to check how far solar/geomagnetic activity interferes with the conception that the signal absorption variability is a fingerprint of planetary wave activity: Fig. 3 (bottom) displays the the geomagnetic disturbance index Ap and its wavelet decomposition for the same time range. The 27 day synodic solar rotation and its first harmonic (13.5 day period) are clearly visible and pronounced during times of increased geomagnetic activity (starting significantly around March 2010 with the new solar cycle) but is not in line with the winter time maxima of low frequency signal ratio periodicities. There is no significant cross correlation between the Ap index and radio propagation periodograms up to the 27 day range (Fig. 4, bottom). However there are clearly common periods with all data in the 27-30 day range. Also noticeable with the Ap wavelet plot are the 5 and 9 day periods. The 9-day periodicities are related to the recurrence 

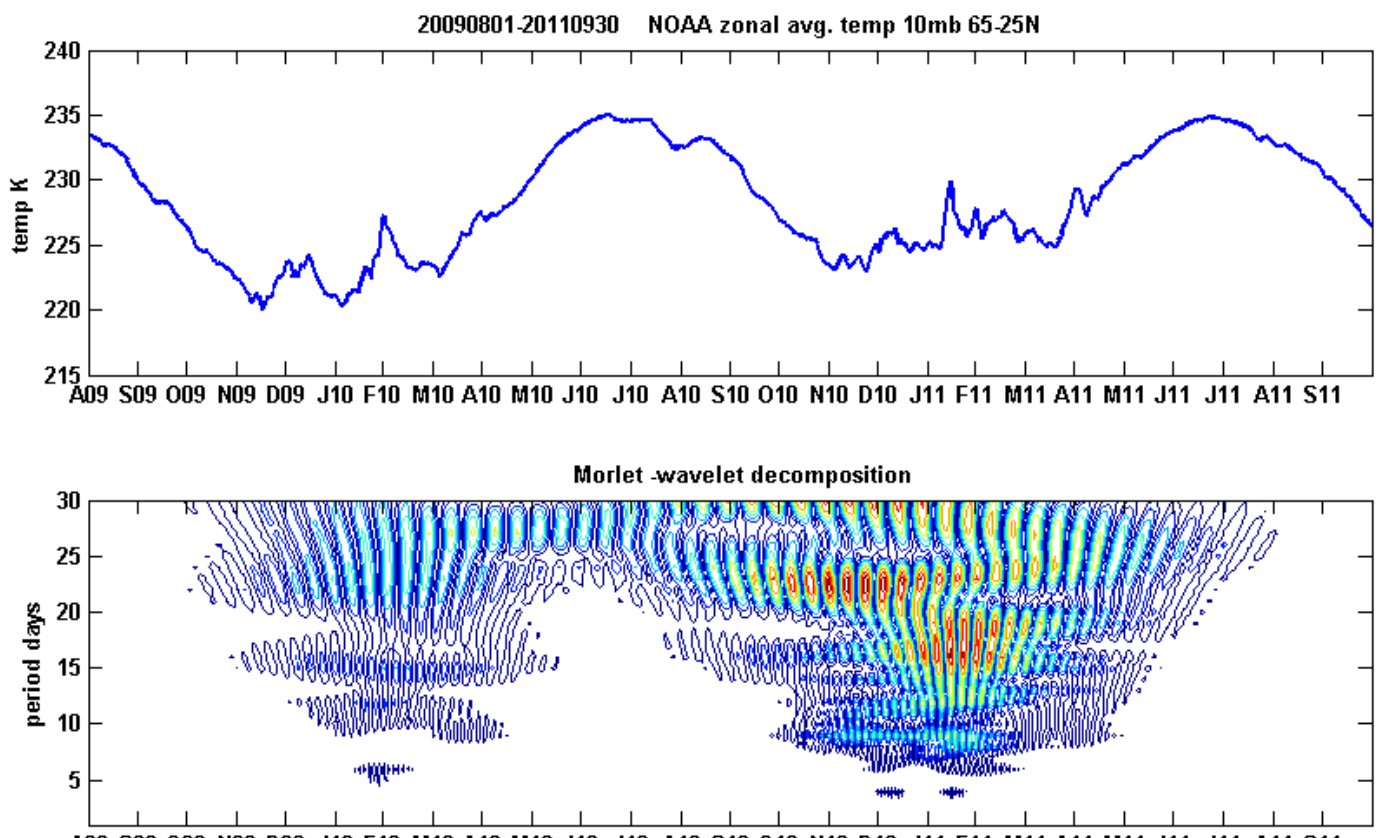

A09 S09 009 N09 D09 J10 F10 M10 A10 M10 J10 J10 A10 S10 010 N10 D10 J11 F11 M11 A11 M11 J11 J11 A11 S11
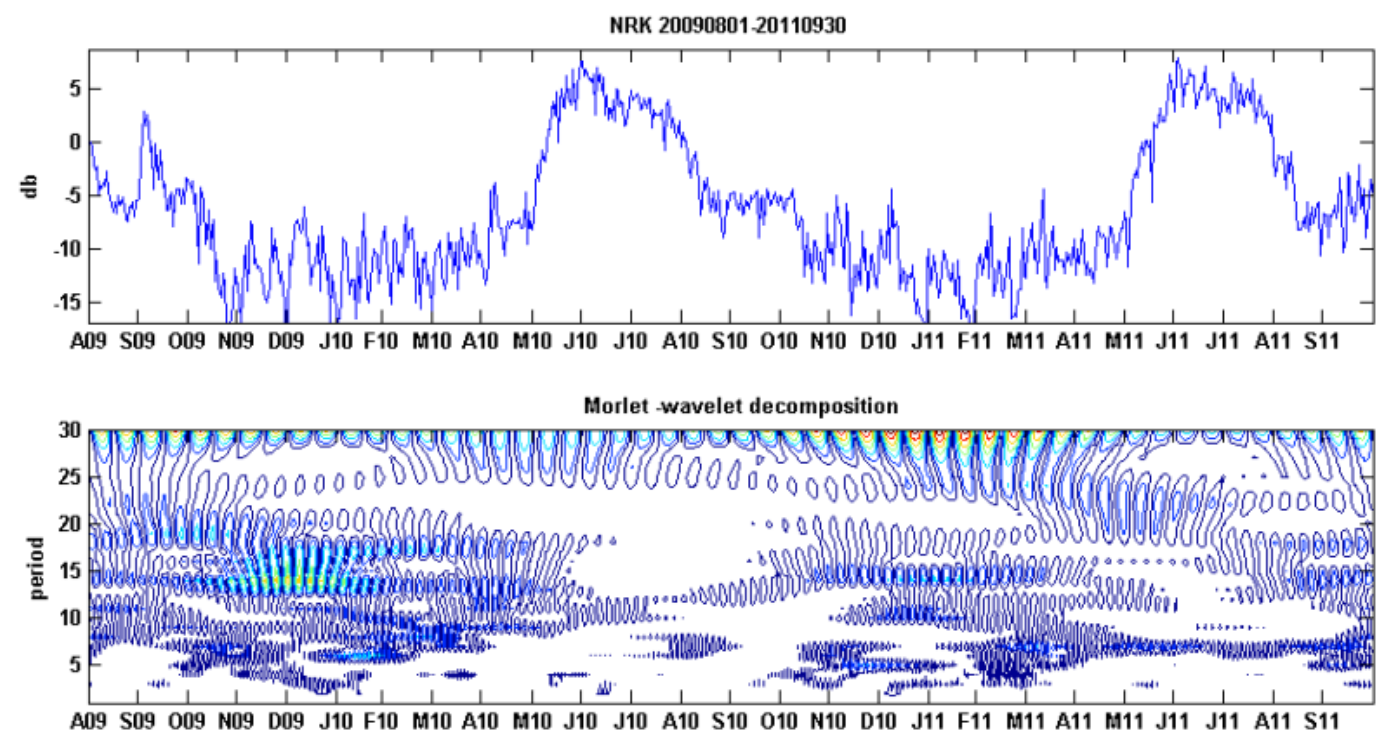

Fig. 2. Time range 1 August 2009 to 30 September 2011, top: $10 \mathrm{hPa}$ (about $30 \mathrm{~km}$ height) zonal mean temperatures for the latitude range 65 to 25 degree north (data from ftp.cpc.ncep.noaa.gov) and the wavelet decomposition. Below: signal amplitude ratio noon/midnight ( $2 \mathrm{~h}$ averages) and wavelet decomposition for the NRK (Iceland) propagation path to $52^{\circ} \mathrm{N} 8^{\circ} \mathrm{E}$.

of fast solar wind streams due to solar coronal holes which are distributed roughly 120 degrees apart in longitude modulating geomagnetic activity and also thermospheric densities (e.g. Lei at al., 2008; Mukhtarov et al., 2010; Emery et al., 2011, we thank one of the referees for pointing us to this result). The 5 day periods around March/April and August/September in 2010 and 2011 roughly coincide with the equinoxes, an effect which is related to the well known semiannual variation of geomagnetic activity with equinoctial maxima (McIntosh, 1959).

\section{Summary and conclusions}

Wavelet analysis - especially using Morlet wave packets - is well suited to localize the intensity of periodicities not only in frequency (period scale) but also in time. Cross correlation 


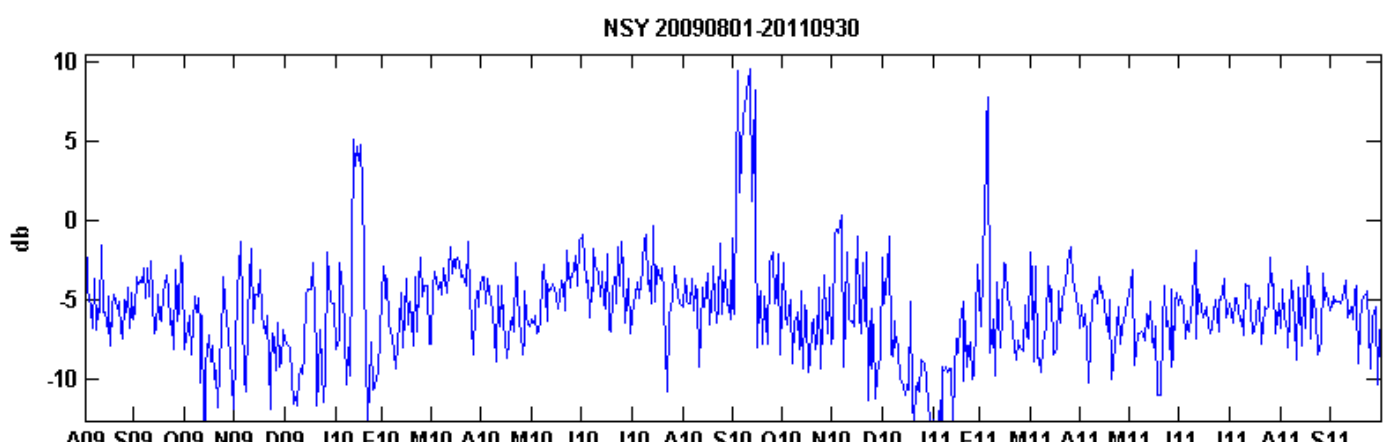

A09 S09 009 N09 D09 J10 F10 M10 A10 M10 J10 J10 A10 S10 010 N10 D10 J11 F11 M11 A11 M11 J11 J11 A11 S11
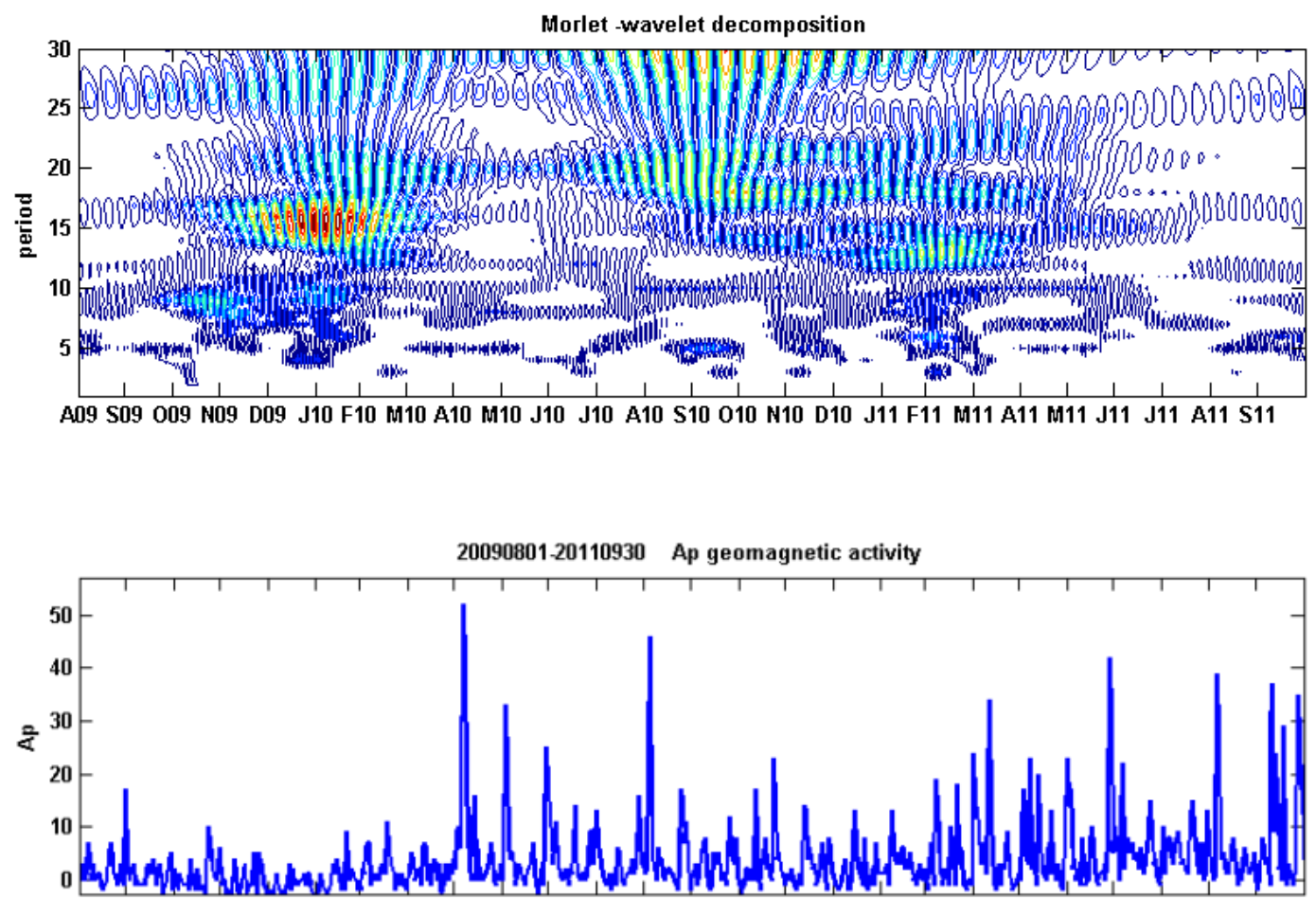

A09 S09 009 N09 D09 J10 F10 M10 A10 M10 J10 J10 A10 S10 010 N10 D10 J11 F11 M11 A11 :111 J11 J11 A11 S11

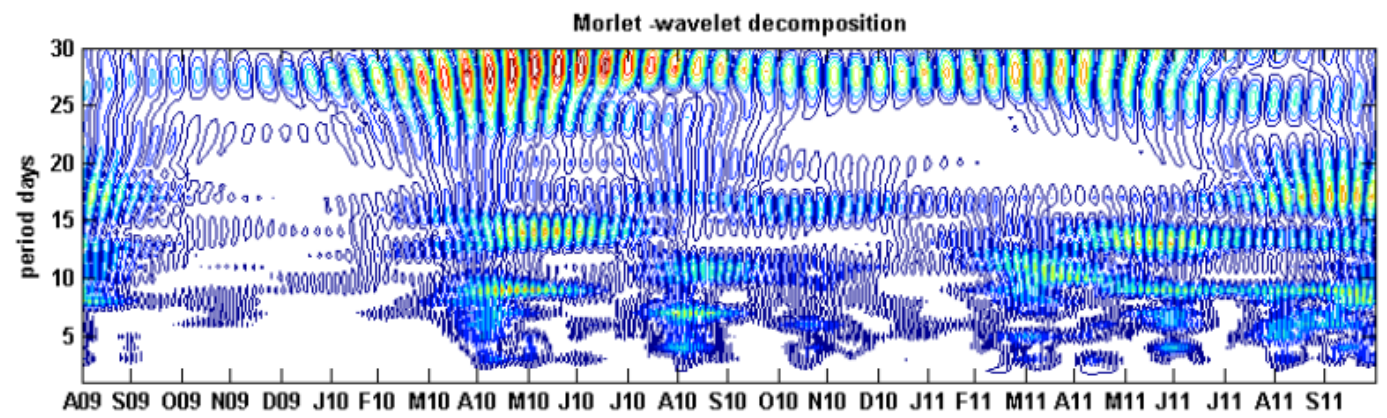

Fig. 3. Time range 1 August 2009 to 30 September 2011, top: signal amplitude ratio noon/midnight ( $2 \mathrm{~h}$ averages) and wavelet decomposition for the NSY (Sicily) propagation path to $52^{\circ} \mathrm{N} 8^{\circ}$ E. Bottom: time series of the geomagnetic Ap index and its wavelet decomposition. The 27 day synodic sun rotation period and its first harmonic (13.5 days) are prominent during times of higher geomagnetic activity. We also note 5 and 9 day periods with the Ap index, see text. 

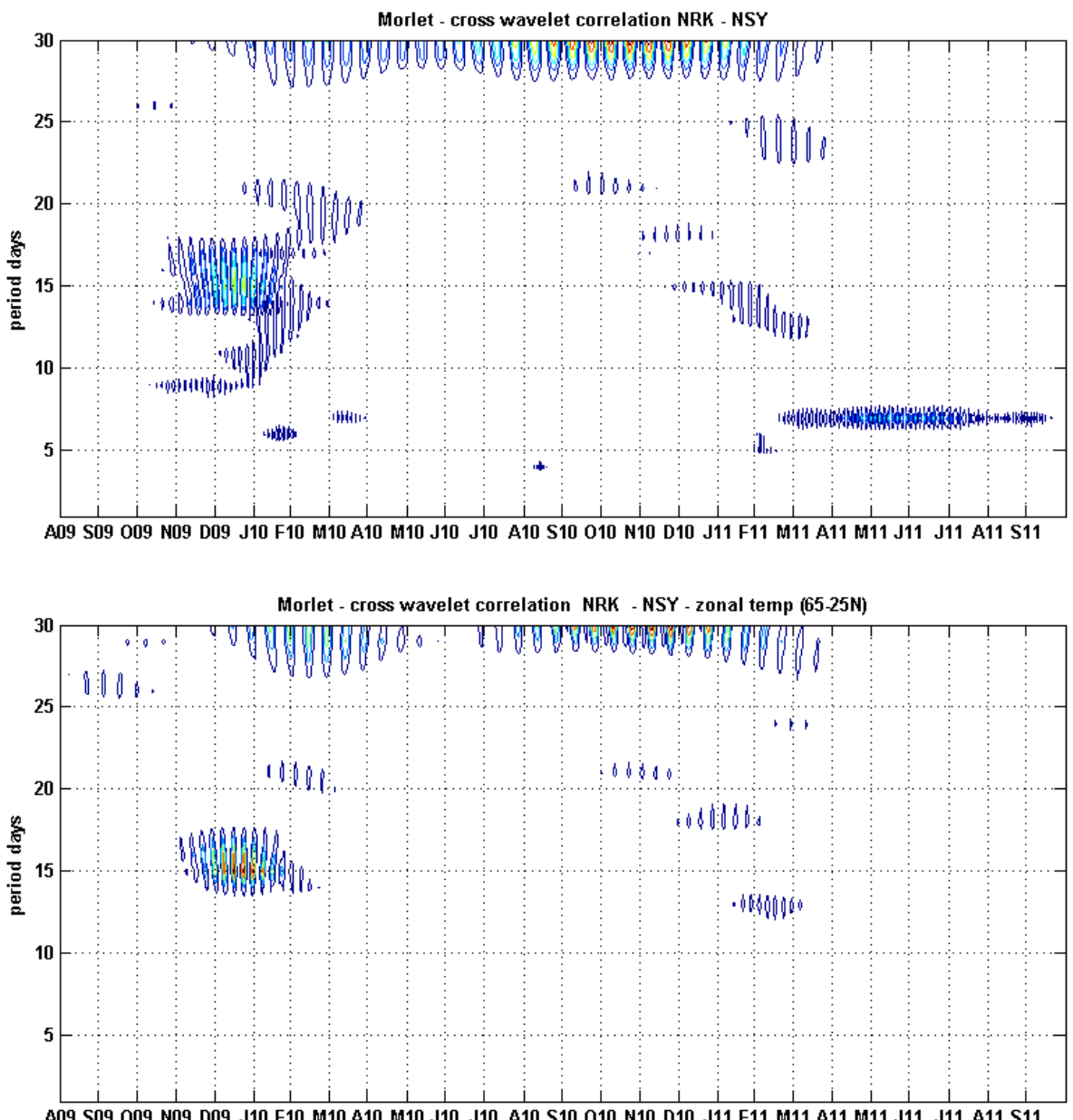

A09 S09 009 N09 D09 J10 F10 M10 A10 M10 J10 J10 A10 S10 010 N10 D10 J11 F11 M11 A11 M11 J11 J11 A11 S11

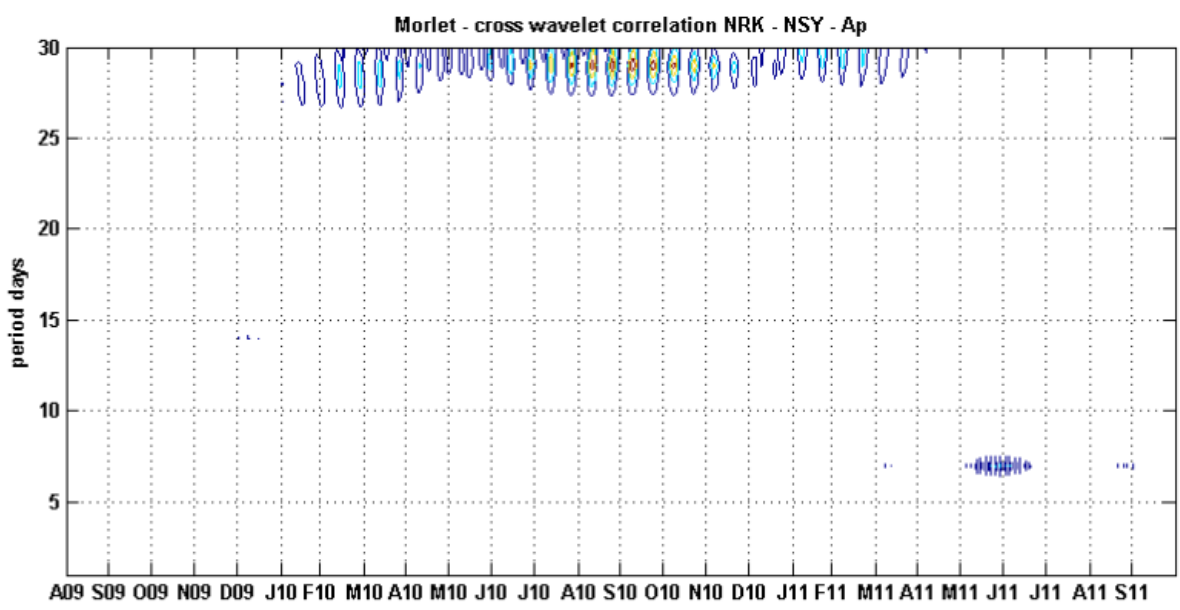

Fig. 4. Time range 1 August 2009 to 30 September 2011: top: cross wavelet analysis of the NRK and NSY transmitter signal day/night ratios. The 7 day period is due to weekly transmitter drop outs. Middle: NRK-NSY-zonal temperature cross correlation. Prominent are the near 16 day period correlations during winter 2009/2011 between the 2 propagation paths as well as with the temperature data. Less significant but nevertheless clear are the near 13 and 18 day period correlations during winter 2010/2011. Bottom: NRK-NSY-Ap cross correlation. There is no significant cross correlation between the Ap index and radio propagation data up to the 27 day range. However there is a clear correlation between all data in the 27-30 day range. 
of the periodograms points to common time ranges of periodicity. The day/night amplitude ratio of $40 \mathrm{kHz}$ low frequency MSK transmitters essentially is an indicator for day over absorption of radiation in the lower ionosphere in the height range $65-85 \mathrm{~km}$. Some $2 \mathrm{yr}$ of monitoring a northern and a southern propagation path to a midlatitude site clearly show periods of 12-17 days (quasi 16 day wave) in the wavelet analyzed data during winter time, not related to solar/geomagnetic activity, together with a strong correlation with seasonal stratosphere temperature changes over the year - more explicitly on the northern path. Traces of periods near 2, 5 and 10 days, presumably also normal planetary modes, can additionally be identified in the radio propagation data (also with the temperature data, however not explicitly correlating in time with the radio data). A modulation of ionospheric parameters, electron density and especially collision frequency via neutral density variations caused by planetary wave activity is endorsed by these results (Lastovicka et al., 1994a; Schmitter, 2011). Recurrent details like the nearly constant level in the amplitude ratios from August to September as well as an assessment of the winter time signal variability with respect to the fingerprint of stratospheric warmings need more data and explanation. Our observation time range (August 2009-September 2011) encompasses part of the long solar minimum and the start of activity within the new solar cycle 24. Future data of our ongoing continuous monitoring will show if and how the absorption behaviour, especially with regard to the day time absorption and its relation to generating planetary waves, will change with increasing solar activity and if other long term trends can be identified (Lastovicka, 2002 reports no sufficiently consistent observational pattern of trends in the lower ionosphere from his long term data). In any case data analysing low frequency MSK signal amplitude ratio measurements prove as an inexpensive additional method for atmosphere and climate research.

Acknowledgements. Topical Editor Matthias Förster thanks Dora Pancheva and Kirsti Kauristie for their help in evaluating this paper.

\section{References}

Emery, B. A., Richardson, I. G., Evans, D. S., Rich, F. J., and Wilson, G. R.: The Sun-Earth Connection Near Solar Minimum: Solar Rotational Periodicities and the Semiannual Variation in the Solar Wind, Radiation Belt, and Aurora, Sol. Phys., 274, 399425, 2011
Fraser, G. J.: The 5-day wave and ionospheric absorption, J. Atmos. Terr. Phys., 39, 121-124, 1977.

Goupillaud, P., Grossman, A., and Morlet, J.: Cycle-Octave and Related Transforms in Seismic Signal Analysis, Geoexploration, 23, 85-102, 1984.

Lauter, E. A., Taubenheim, J., and Von Cossart, G.: Monitoring middle atmosphere processes by means of ground-based lowfrequency radio wave sounding of the D-region, J. Atmos. Terr. Phys., 46, 775-780, 1984.

Lastovicka, J.: Trends in planetary wave activity in the upper middle atmosphere inferred from the nighttime LF radio wave absorption measurements, Stud. Geophys. Geod., 38, 206-212, 1994.

Lastovicka, J.: Long-term changes and trends in the lower ionosphere, Phys. Chem. Earth, Parts A/B/C 27, 497-507, 2002.

Lastovicka, J., Ebel, A., and Ondraskova, A.: On the transformation of planetary waves of tropospheric origin into waves in radio wave absorption in the lower ionosphere, Stud. Geophys. Geod., 38, 71-81, doi:10.1007/BF02296254, 1994a.

Lastovicka, J., Fiser V., and Pancheva, D.: Long-term trends in planetary wave activity (2-15 days) at $80-100 \mathrm{~km}$ inferred from radio wave absorption, J. Atmos. Terr. Phys., 56, 893-899, 1994b.

Lei, J., Thayer, J. P., Forbes, J. M., Sutton, E. K., and Nerem, R. S.: Rotating solar coronal holes and periodic modulation of the upper atmosphere, Geophys. Res. Lett., 35, L10109, doi:10.1029/2008GL033875, 2008.

Pogoreltsev, A. I., Kanukhinaa, A. Yu., Suvorova, E. V., and Savenkovaa, E. N.: Variability of planetary waves as a signature of possible climatic changes, J. Atmos. Sol.-Terr. Phy., 71, 1529-1539, 2009.

McIntosh, D. H.: On the annual variation of magnetic disturbance, Phil. Trans. Roy. Soc. Lond., 251, 525-552, 1959.

Mukhtarov, Pl., Andonov, B. ,Borries, C., Pancheva, D., and Jakowski, N.: Forcing of the ionosphere from above and below during the Arctic winter of 2005/2006, J. Atmos. Sol.-Terr. Phys., 72, 193-205, doi:10.1016/j.jastp.2009.11.008, 2010.

Schmitter, E. D.: Remote auroral activity detection and modeling using low frequency transmitter signal reception at a midlatitude site, Ann. Geophys., 28, 1807-1811, doi:10.5194/angeo-281807-2010, 2010.

Schmitter, E. D.: Remote sensing planetary waves in the midlatitude mesosphere using low frequency transmitter signals, Ann. Geophys., 29, 1287-1293, doi:10.5194/angeo-29-1287-2011, 2011.

Schwentek, H.: Wave-like structures in the variation of ionospheric absorption, J. Atmos. Terr. Phys., 36, 1173-1178, 1974.

Volland, H.: Atmospheric tidal and planetary waves, Kluwer, 1988. 